\title{
Recomendaciones para optimizar el éxito en reimplante de dedos
}

\section{Recommendations to Optimize the Success of Finger Replantations}

\author{
Luis Landin-Jarillo ${ }^{1} \quad$ David Gonzalez-Peinado ${ }^{1} \quad$ Javier Alonso-Sanz ${ }^{1} \quad$ Carlos Villar-Yus ${ }^{1}$ \\ Cesar Casado-Sanchez ${ }^{1}$ \\ ${ }^{1}$ Fundación FiBHULP-IdiPaz, Servicio de Cirugía Plástica y Reparadora, \\ Hospital Universitario La Paz, Madrid, España \\ Address for correspondence Dr. Luis Landín Jarillo, PhD, Servicio de \\ Cirugía Plástica, Paseo de la Castellana 261, 28046 Madrid, España \\ Rev Iberam Cir Mano 2019;47:115-123. \\ (e-mail: Ilandin@salud.madrid.org).
}

\section{Resumen \\ Palabras clave \\ - reimplante \\ - revascularización \\ - microcirugía \\ - dedo \\ - amputación}

Los traumatismos con amputación o desvascularización de los dedos de la mano son lesiones complejas que precisan la derivación a unidades de referencia. El objetivo del reimplante es un resultado funcional y estético, y depende del traumatismo, del paciente y del cirujano. El éxito observado en reimplantes y revascularizaciones ha alcanzado el $89,5 \%$ en nuestra serie de 130 pacientes entre los años 2006 y 2019. La valoración inicial del paciente y de la parte amputada tienen repercusión directa en la indicación y el resultado final del reimplante. El desempeño de las técnicas de microcirugía es esencial para el éxito del procedimiento. En esta revisión, damos a conocer los diagnósticos prácticos así como la secuencia para la ejecución del reimplante desde antes de la llegada del paciente a quirófano, y proponemos una serie de detalles técnicos para ayudar al lector a conseguir el éxito de sus reimplantes.

\begin{abstract}
Injuries involving amputation or devascularization of the fingers are complex lesions which require that the patient is brought to a specialized and reference center. The ultimate goal of replantation is a good functional and cosmetic result, which will be conditioned by trauma-dependent factors, the patient and the surgeon. The success rate found in replantations and revascularizations is currently around $89,5 \%$ in our series of 130 patients between 2006 and 2019. An accurate initial assessment of the patient and the amputated part are critical and have a direct impact on the indication

Keywords

- replant

- finger

- digit

- microsurgery and the final outcome of the replantation. The performance in microsurgery techniques is essential for the success of the procedure. In this review we present practical diagnoses as well as the sequence of replantation, starting even before the patient reaches the operating room. We also suggest a series of technical tips to help the reader succeed in replantation surgery.
\end{abstract}

\section{Introducción}

Los traumatismos complejos y las amputaciones de los dedos de la mano afectan de manera importante a los pacientes en la esfera funcional-laboral, pero también son una importante causa de estigma estético por la pérdida de la corporalidad. Tradicionalmente la revisión del muñón de amputación se ha received

September 22, 2019

accepted

September 30, 2019
DOI https://doi.org/

10.1055/s-0039-3399513. ISSN 1698-8396.
Copyright $\odot 2019$ Thieme Revinter

Publicações Ltda, Rio de Janeiro, Brazil
License terms

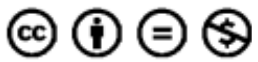


realizado en centros sin dedicación específica. Sin embargo, la demanda de un mejor resultado, con independencia del tiempo de baja laboral, ha aumentado notablemente en los últimos años. 1

La atención en centros de referencia para esas lesiones presenta mayor tasa de indicación del reimplante, mayor éxito en la cirugía reparadora, disminución de los tiempos de ingreso hospitalario, menor coste económico y mayor planificación de procedimientos secundarios. ${ }^{2-4}$ El dominio de las técnicas microquirúrgicas permite indicar el reimplante disminuyendo de forma notable las secuelas por amputación, a la vez que aumenta la satisfacción de los pacientes con el tratamiento recibido. ${ }^{5-7}$ Es fundamental no confundir el éxito de las anastomosis vasculares con el éxito del resultado funcional. La formación en microcirugía solamente facilita entrar en este apasionante mundo de la reconstrucción microquirúrgica. ${ }^{7}$ Pero la indicación del reimplante es el primer paso en un complejo camino en el que la experiencia en cirugía secundaria, psicología del paciente y rehabilitación específica facilita las posibilidades de llegar a la meta funcional que nos proponemos.

En esta revisión presentamos los detalles organizativos y técnicos aprendidos en los últimos 13 años dedicados a la cirugía de reimplante de miembro superior. Este trabajo se fundamenta en la realización de reimplantes en 130 pacientes con un éxito del $89,5 \%$.

\section{Nivel y Grado de Amputaciones Digitales}

Para describir las lesiones por amputación y establecer las probabilidades de reimplante debemos especificar el grado y el nivel anatómico de la lesión. Según el grado de afectación se pueden dividir en desvascularización y amputación.

En las lesiones por desvascularización existe una detención en el flujo sanguíneo a la zona distal a la lesión, mientras que las amputaciones son lesiones en las que la parte lesionada se encuentra totalmente separada del cuerpo. La diferencia es importante ya que los procedimientos de revascularización no requieren la reparación de venas y suelen ser más sencillos, rápidos y con mayor probabilidad de éxito. Por el contrario, los procedimientos de reimplante son más complejos, más largos y tienen mayor riesgo de fracaso debido sobre todo a la congestión venosa.

Un caso especial son las amputaciones en continuidad, en las que la parte lesionada se encuentra conectada por medio de alguna estructura anatómica, típicamente un puente cutáneo, tendón o un nervio colateral. La conexión por puentes cutáneos disminuyen notablemente el riesgo de congestión venosa. La - Figura 1 muestra ejemplos de desvascularización, amputación y amputación en continuidad.

El nivel de la amputación se especifica atendiendo a la zona del tendón flexor superficial donde tuvo lugar la lesión (-Figura 2). Otra situación especial son las lesiones en dedo de anillo o degloving, en las que se produce un deguantamiento de un dedo a nivel subcutáneo (- Figura 3). Esas lesiones se deben describir según la clasificación de Urbaniak, que hemos modificado para añadir valiosa información sobre indicación microquirúrgica en la - Tabla $1 .^{8}$

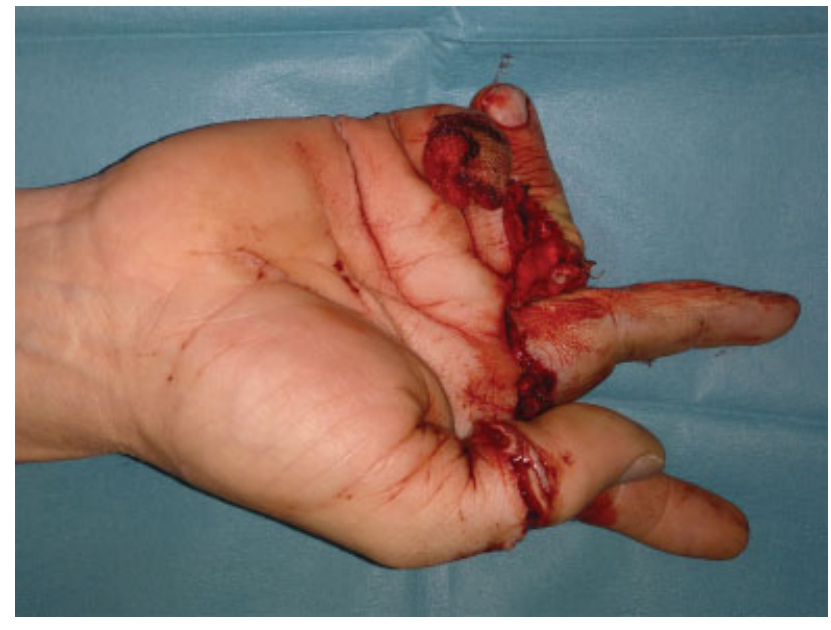

Fig. 1 La imagen muestra diferentes lesiones digitales. El primer dedo muestra un herida radial. El segundo presenta desvascularización, mientras que el tercero y el cuarto dedos presentan amputaciones en continuidad. El quinto dedo presenta amputación completa.

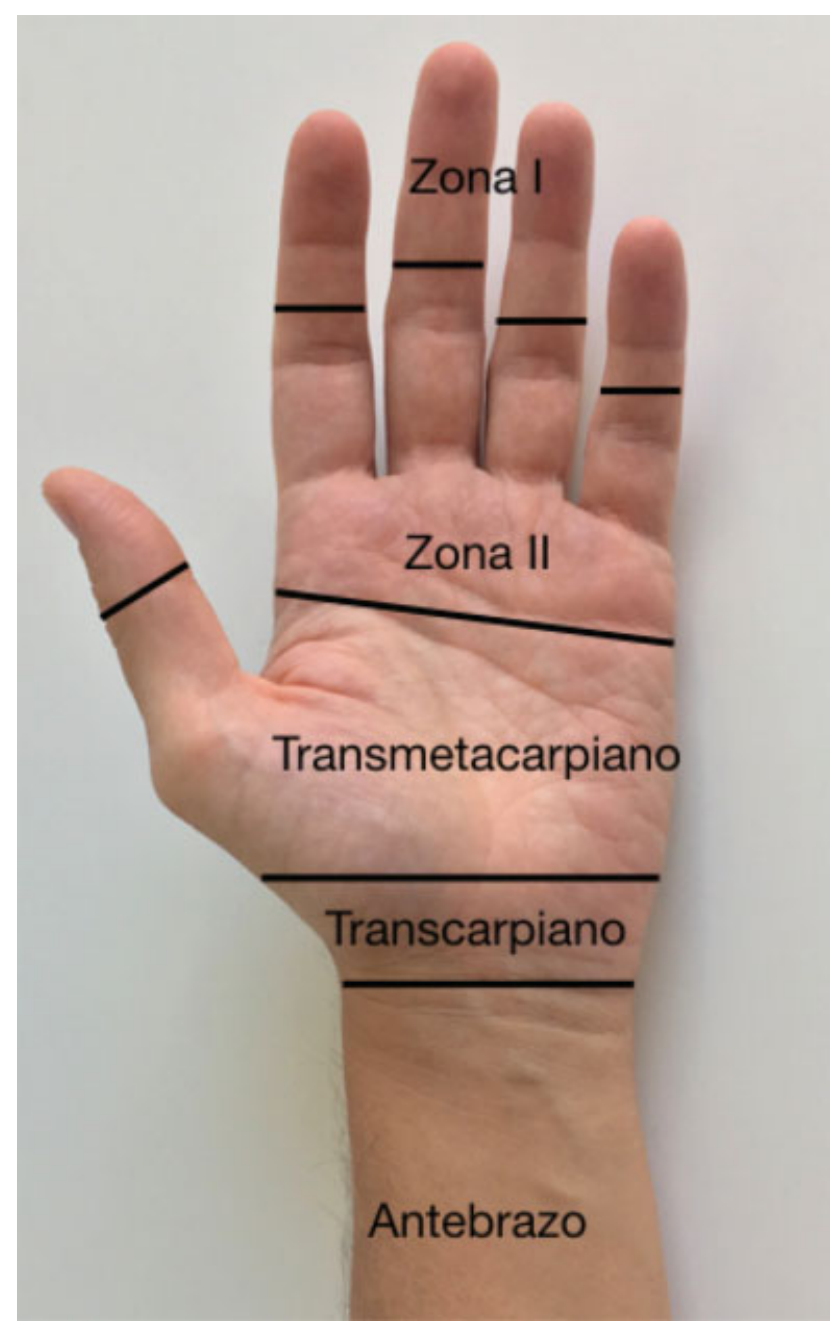

Fig. 2 Clasificación práctica de las zonas de lesión.

\section{Mecanismos de Amputación, Lesión Vascular e Indicación de Reimplante}

Los mecanismos de amputación contusivos y avulsivos son los más frecuentes, siendo el mecanismo por aplastamiento 


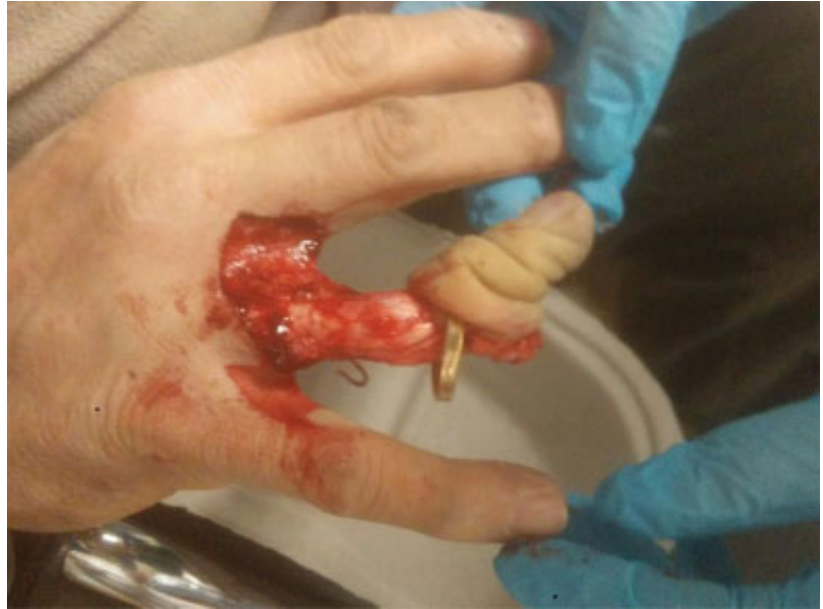

Fig. 3 Ejemplo de lesión en dedo de anillo o degloving tipo II de la clasificación de Urbaniak.

menos frecuente. ${ }^{3}$ El mecanismo de guillotina, a pesar de ser la técnica más favorable y funcional, apenas ocurre. El mecanismo lesional por sí solo no contraindica la cirugía, pero nos informa sobre la dificultad técnica del reimplante, las posibles complicaciones y futuro resultado funcional (-Tabla 2).

Dos factores que deben valorarse para indicar el reimplante son la lesión vascular en la parte amputada y los resultados funcionales esperados. La lesión vascular de la parte amputada debe valorarse bajo magnificación óptica. La presencia de ramas arteriales rotas en la arteria colateral del dedo nos informa del alcance de la lesión traumática. La arteria colateral debe desbridarse hasta alcanzar una zona en la que no haya ramas rotas por el traumatismo ni hematomas en la pared vascular. Además, la salida telescopada de la íntima por el cabo arterial indica lesión severa. Por tanto, se debe continuar el desbridamiento hacia una zona más alejada de la lesión.

La realización de anastomosis en zonas de arteria lesionada conduce a la formación de trombo blanco plaquetario y fracaso de la anastomosis. Clínicamente se observa de forma típica un latido arterial longitudinal (en el sentido direccional del vaso), lo que indica un empuje del latido sobre el trombo. Las anastomosis funcionantes en cambio presentan un latido transversal, por dilatación y contracción del vaso en cada latido sanguíneo.

Como norma general son reimplantables aquellas partes amputadas en las que haya una anatomía reconocible. Los resultados funcionales esperables dependen de la propia
Tabla 2 Factores pronósticos en reimplantes de miembro superior

\begin{tabular}{|l|l|}
\hline $\begin{array}{l}\text { Factores de } \\
\text { buen pronóstico }\end{array}$ & Factores de mal pronóstico \\
\hline $\begin{array}{l}\text { Mecanismo } \\
\text { tipo guillotina }\end{array}$ & Avulsión o aplastamiento \\
\hline Edad joven & Isquemia caliente $>6 \mathrm{~h}$ \\
\hline Nivel distal & Isquemia fría $>12 \mathrm{~h}$ \\
\hline & Multinivel \\
\hline & Pérdida masiva de hueso \\
\hline & Pérdida nerviosa \\
\hline & Edad avanzada \\
\hline & Múltiples articulaciones afectadas \\
\hline & Mal candidato para la inmovilización \\
\hline & Problemas psicosociales \\
\hline & Mala disposición a la rehabilitación \\
\hline
\end{tabular}

lesión, del paciente en cuestión y del equipo quirúrgico y su experiencia. En nuestro caso contraindicamos el reimplante cuando no hay una anatomía reconocible.

Las lesiones avulsivas, además de requerir frecuentemente la realización de by-pass, suelen tener pérdida de ciertas zonas anatómicas y merecen una consideración especial, ya que suelen necesitar cirugía secundaria para mejorar la función. ${ }^{9,10}$ Las amputaciones por aplastamiento y las avulsiones se asocian a una mayor extensión del daño de la capa íntima y de las ramas vasculares, por lo que pueden presentar mayores dificultades de reimplante incluso para cirujanos expertos.

Los reimplantes o revascularizaciones de dedos de anillo pueden cursar con buenos resultados funcionales, por lo que se recomienda intentar el reimplante en centros especializados. ${ }^{11}$ Nuestras indicaciones de reimplante se muestran en la -Tabla 3.

Tabla 3 Indicaciones de reimplante

\begin{tabular}{|l|}
\hline Anatomía reconocible de la parte amputada \\
\hline Amputación de dedo largo en zona I \\
\hline Amputación multidigital en zona II \\
\hline Amputación de pulgar \\
\hline Amputación en edad pediátrica \\
\hline
\end{tabular}

Tabla 1 Clasificación de las lesiones por degloving y su repercusión quirúrgica

\begin{tabular}{|l|l|l|l|l|l|}
\hline Tipo & $\begin{array}{l}\text { Amputación c } \\
\text { ompleta }\end{array}$ & Lesión cutánea & Lesión ósea & Circulación & Indicación quirúrgica \\
\hline I & No & Avulsión cutánea & $+/-$ & Correcta & Reparación cutánea \\
\hline II & No & $\begin{array}{l}\text { Avulsión cutánea y } \\
\text { neurovascular }\end{array}$ & $+/-$ & Inadecuada & By-pass en arterias \pm by-pass venas \\
\hline III & Sí & Amputación interfalángica & + & Inadecuada & $\begin{array}{l}\text { By-pass en arterias } \pm \text { by-pass } \\
\text { venas }+ \text { artrodesis interfalángica distal }\end{array}$ \\
\hline
\end{tabular}


En relación a las características propias del paciente, son peores candidatos al reimplante aquellos pacientes de edad avanzada, pluripatológicos y fumadores. ${ }^{12}$ Los pacientes en una situación psiquiátrica inestable y los pacientes con trastornos trombofílicos conocidos podrían tener contraindicación para el reimplante.

\section{Secuencia del Reimplante}

\section{Primera Atención y Derivación a Hospital de Referencia}

Durante la primera atención en Urgencias, se realiza la exploración del paciente y de la parte amputada, especificando el nivel, grado y mecanismo de la lesión. Es importante evitar utilizar torniquetes o ligaduras, así como evitar la exploración instrumental del muñón o de la parte amputada, lo cual puede lesionar estructuras vasculonerviosas y poner en riesgo el reimplante. Un vendaje compresivo local es suficiente. Después se procederá a la derivación a una unidad de referencia en reimplantes.

Conviene avisar al hospital receptor para confirmar la aceptación del caso. No recomendamos traslados de macrorreimplantes entre ciudades distantes dado el riesgo de compromiso hemodinámico para el paciente. La amputación nunca debe distraernos y enmascarar otras posibles lesiones de mayor gravedad y/o riesgo vital, asegurando el estado hemodinámico del paciente previo a la indicación de reimplante.

Durante el transporte de la parte amputada hay que evitar congelación de la misma. Para ello se envuelve la parte amputada en gasas húmedas y posteriormente se introduce en una bolsa estanca, que a su vez se introduce en una bolsa con hielos y agua.

\section{Llegada a Hospital de Referencia y Preparación del Paciente}

Los estudios radiográficos deberán realizarse en el hospital que va a realizar el tratamiento definitivo antes de acudir a quirófano. Conviene realizar radiografías del paciente y de la parte amputada para determinar las lesiones óseas asociadas. Además, es conveniente evitar cualquier maniobra anestésica antes de la valoración definitiva por el equipo de reimplante, asegurando una exploración fidedigna, así como la adecuada transmisión de información y el consentimiento de la cirugía y los objetivos perseguidos en la misma. El procedimiento de reimplante se realiza bajo anestesia general, sondaje vesical, soporte térmico y protección de prominencias óseas.

\section{Preparación de Parte Amputada}

Podremos comenzar la evaluación quirúrgica y preparación de la parte amputada (ej.: desbridamiento y referencia de estructuras) mientras el paciente es estabilizado, trasladado o anestesiado. El tiempo ahorrado permitirá reducir el tiempo de anestesia y maximizará la eficiencia de la cirugía. ${ }^{13}$ Esa maniobra se realiza en una mesa de Mayo con material quirúrgico y de microcirugía, sedas $6 / 0$ para marcaje de estructuras y clips vasculares. Como medio de magnificación óptica recomendamos lupas de $\times 3.5$ o idealmente de $\times 4.5$ aumentos o como último recurso en esa fase el microscopio óptico, debido a sus notables limitaciones de ajuste de posición de la cabeza.

Comenzamos la secuencia del reimplante con el lavado de suero fisiológico de la parte amputada, extracción de cuerpos extraños y desbridamiento de bordes cutáneos y tejidos desvitalizados. No es posible realizar un reimplante por la misma herida de amputación ya que el riesgo de estar trabajando sobre zonas de lesión vascular es alto y podrían comprometer el éxito microvascular. Así que por medio de incisiones laterales podemos alejarnos de la zona de traumatismo hasta encontrar vasos sanos (-Figuras 4 y $\mathbf{5}$ ).

La incisión cutánea lateral y la disección de las arterias digitales deben continuar hasta llegar fuera de la zona de lesión. El desbridamiento de los vasos debe ser agresivo hasta encontrar vasos sanos, cilíndricos y sin ramas rotas. En cambio, los muñones nerviosos se deben preparar con un desbridamiento conservador. Además los nervios se deben separar de las arterias, y en ocasiones es necesario sacrificar pequeñas ramitas laterales. Todos los cabos deben ser marcados en su extremo con una ligadura de seda de 6/0.

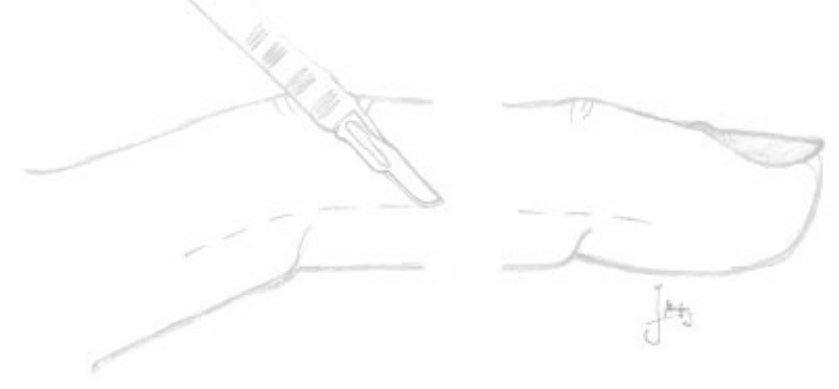

Fig. 4 Ejemplo de incisiones laterales axiales para identificar pedículos neurovasculares en el muñón y en la parte amputada.

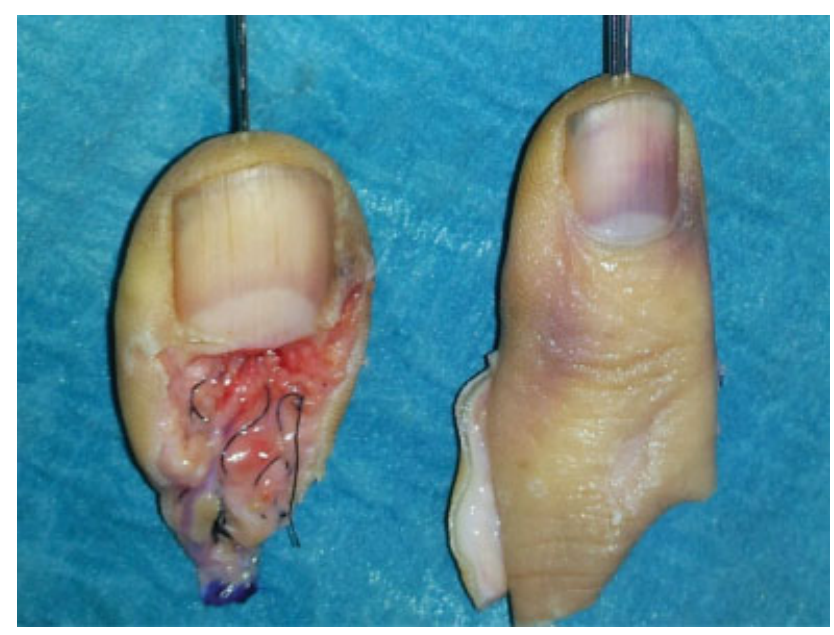

Fig. 5 Marcaje de las estructuras vasculonerviosas con seda de 6/0 e introducción de dos agujas de Kirschner en cada dedo. También se observa incisión lateral en el dedo. 


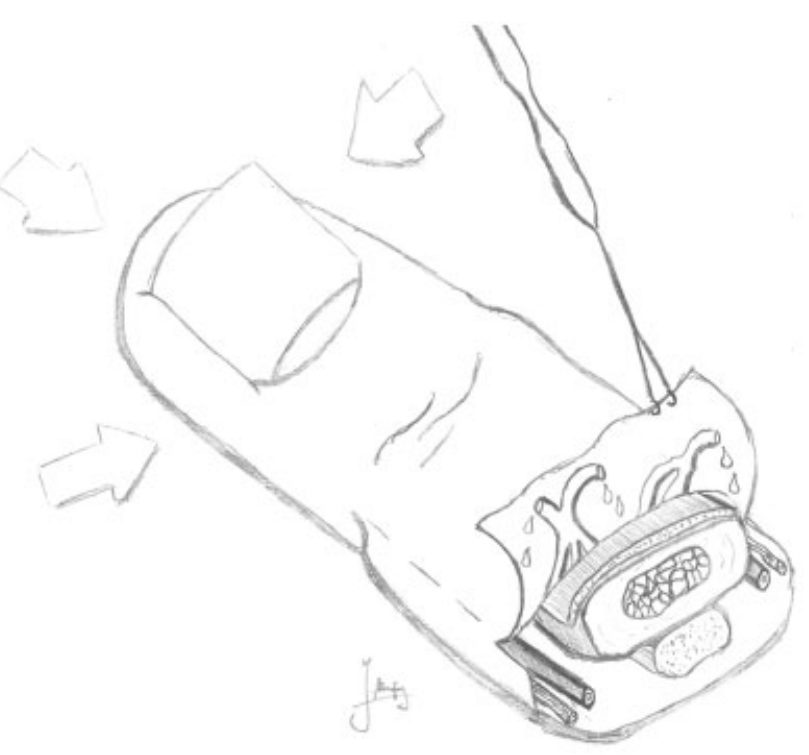

Fig. 6 Preparación de segmento amputado. Elevación del colgajo cutáneo dorsal con venas dorsales. La exanguinación por compresión del pulpejo del dedo facilita la identificación de las venas.

Seguidamente se eleva un colgajo cutáneo del dorso que contenga la grasa y las venas del dorso por encima del aparato extensor (-Figura 6). Por lo general, la exanguinación por compresión del pulpejo del dedo facilita el retorno de sangre por esas venas y ayuda a la identificación de los cabos venosos. La lesión vascular en las venas casi siempre ocurre en las bifurcarciones, por lo que es necesario desbridar más longitud para alcanzar un segmento cilíndrico sano. Igualmente los cabos venosos se marcan con ligadura de seda 6/0 para facilitar su posterior identificación y reparación (- Figuras 5 y $\mathbf{6}$ ).

Seguidamente se diseca el aparato extensor y el tendón flexor profundo (FDP), separándolos de la falange con un disector romo. En el cabo amputado de FDP se realiza una sutura de Kessler modificada con prolene de $4 / 0$ dejando los cabos preparados para su sutura recíproca luego en el muñón. Después se realiza el desbridamiento óseo (- Figura 7), recomendando un acortamiento óseo suficiente que prevenga el uso de by-pass en las anastomosis venosas. La osteosíntesis se puede preparar con agujas de Kirschner (-Figuras 5 y 7) o con cerclajes de hilo de acero cruzados $90^{\circ}$. Las agujas de Kirschner se introducen desde el propio canal medular expuesto, de forma retrógrada y en sentido inverso, de tal manera que dejamos la punta de la aguja preparada para su inserción en la zona de amputación.

La preparación de la parte amputada suele requerir unos 30 minutos por cada dedo y, una vez finalizado, el paciente ya se encuentra en quirófano y anestesiado y puede comenzar la segunda fase del reimplante.

\section{Preparación del Muñón Proximal}

La preparación del muñón proximal puede realizarse de manera simultánea o tras la preparación de las partes amputadas, dependiendo de la disponibilidad de dos o un solo equipo quirúrgico respectivamente. La preparación comienza con la aplicación de manguito de isquemia a 250

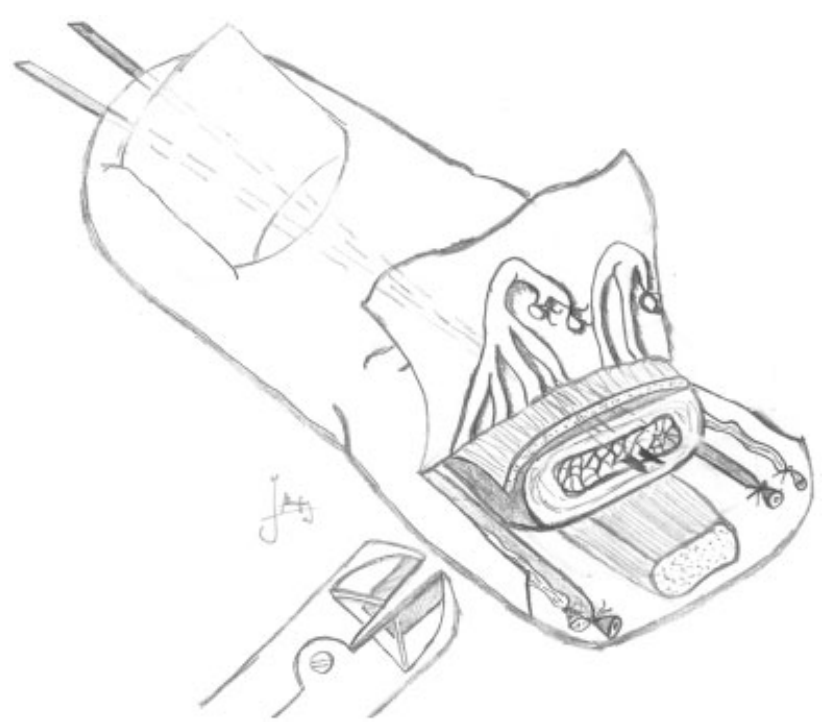

Fig. 7 Preparación de segmento amputado. Disección y marcaje con ligaduras de seda $6 / 0$ de los pedículos. Elevación de colgajo cutáneo dorsal para identificar y marcar las venas del dorso. Desbridamiento/acortamiento óseo mediante el empleo de cizalla. Preparación de la osteosíntesis mediante la introducción de 2 agujas de Kirschner.

mmHg previa exsanguinación por elevación del miembro durante un minuto. El manguito de isquemia se puede mantener hasta 2 horas. En caso de alcanzar el tiempo máximo, recomendamos soltar isquemia y esperar al menos 10 minutos antes de volver a aplicar el torniquete.

El muñón proximal se aborda mediante incisiones laterales hasta identificar los pedículos neurovasculares en zona fuera de la lesión (- Figura 8). Se debe disecar la arteria proximalmente hasta encontrar un segmento sin los signos

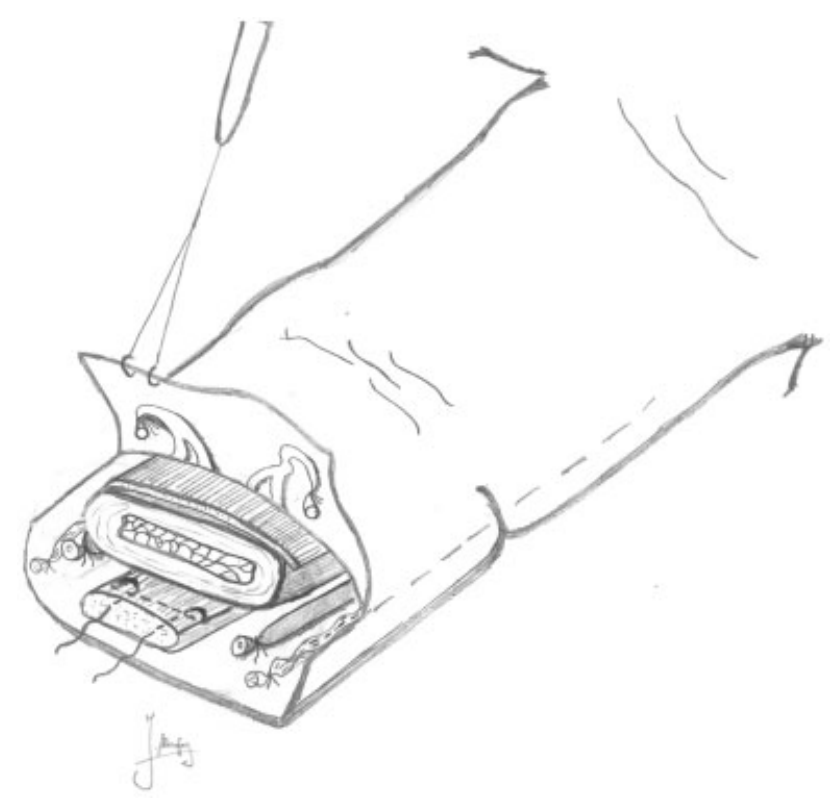

Fig. 8 Preparación de muñón de amputación. Disección y marcaje con ligaduras de seda 6/0 de los pedículos. Elevación de colgajo cutáneo dorsal para identificar y marcar las venas del dorso. Preparación de la tenorrafia de tendón flexor profundo en cabo proximal. 
traumáticos mencionados anteriormente. Según avanza la disección, se deben clipar las ramas arteriales para evitar el sangrado por una de ellas, lo cual produciría un "espasmo de rama insatisfecha". El espasmo de rama insatisfecha produce una isquemia arterial sin resolución espontánea. Las estructuras se marcan con ligaduras de seda $6 / 0$.

Seguidamente se recupera el tendón FDP y se dejan pasados dos cabos en forma de sutura de Kessler modificada que se completarán con su recíproco cuando se reimplante el dedo. En la zona dorsal se eleva el colgajo por encima del aparato extensor y se identifican las venas del dorso, que se disecan hasta alcanzar una zona libre de lesión. Por último, se desbrida la falange a lo largo de $0,5 \mathrm{~cm}$ generando un acortamiento óseo (- Figura 8). La preparación del muñón suele requerir de unos 45 minutos por dedo.

\section{Colocación de la Parte Amputada y Reparación de Estructuras}

Finalmente se coloca el dedo en su posición (-Figura 9) cuidando de forma importante que no se produzca una malrotación axial ni malposición, verificando en la medida de lo posible que las uñas tengan la orientación más parecida posible. Las agujas de Kirschner se pasan hacia la base de la falange, sin necesidad de atravesar la articulación (- Figura 10). El cirujano sostiene el dedo para asegurar la alineación axial mientras el ayudante pasa las agujas.

Una vez estabilizado el dedo se prona la mano para comenzar las reparaciones dorsales. El aparato extensor se

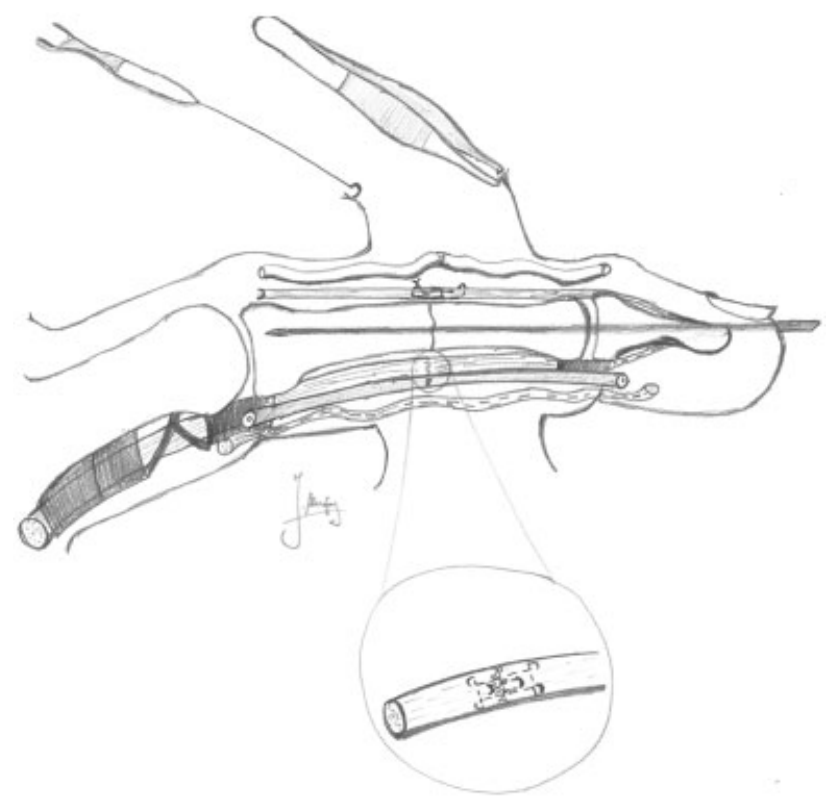

Fig. 9 Implantación del dedo amputado. Osteosíntesis de fractura mediante el paso de 2 agujas de Kirschner. Tenorrafia extensora con puntos en U. Anastomosis microquirúrgica de venas dorsales (la tracción suave de la piel dorsal por parte del ayudante con una pinza puede ayudar a aproximar las venas dorsales distales al nivel de amputación). Tenorrafia flexora (detalle ampliado) mediante sutura tipo Kessler modificado de 2 suturas, puntos en $U$ y epitenorrafia tipo Silfverskiöld. Anastomosis arterial primaria termino-terminal y neurorrafia epineural primaria de nervios colaterales.

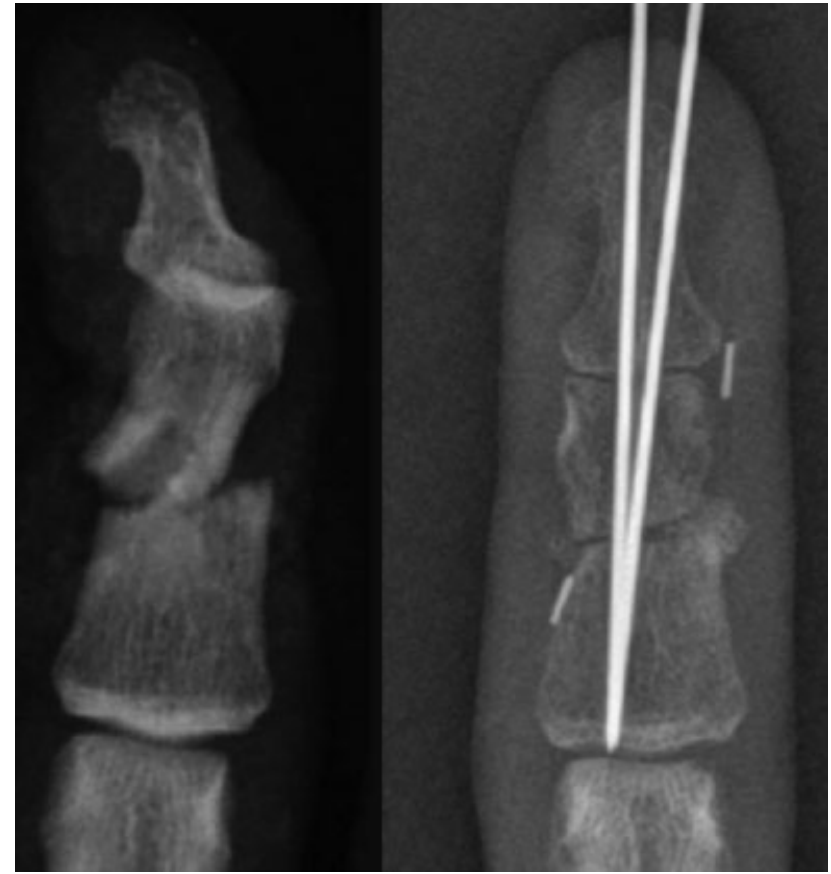

Fig. 10 La radiografía muestra lesión esquelética de un reimplante de dedo índice. A la derecha se muestra acortamiento óseo y estabilización con 2 agujas de Kirschner de 1.0-mm.

debe suturar con puntos en $U$ de hilo monofilamento $4 / 0$. Seguidamente se reparan al menos dos venas. Normalmente el acortamiento óseo permite esas reparaciones sin necesidad de by-pass. La luz vascular debe observarse bajo el microscopio y debe ser fácil ver su interior tras inundar el campo con solución heparinizada (heparina sódica 5000 UI en $100 \mathrm{ml}$ de suero fisiológico). Si la luz vascular presenta flecos o no es cilíndrica, se debe desbridar hasta lograr un cilindro perfecto. La maniobra se repite en la vena receptora previa colocación de un clamp microvascular que se mantendrá durante la sutura. Por lo general, la sutura vascular se realiza con hilo de 9/0 o 10/0 montado sobre aguja cilíndrica de $75 \mu \mathrm{m}$. Una vez reparadas las venas, se sutura el dorso holgadamente con hilo 4/0 (en niños puede utilizarse $5 / 0$ o 6/0) y puede supinarse el miembro para comenzar las reparaciones volares.

Por la parte volar se completa primero la sutura del tendón FDP reuniendo las dos suturas tipo Kessler con 2 cabos $4 / 0$, que se complementa con 2 puntos en $U$ 4/0 y una sutura epitendinosa tipo Silfverskiöld 6/0.

Después se repara al menos una de las arterias o, a ser posible las dos. Aún trabajando en isquemia, es posible obtener reflujo de sangre en la arteria que le llega desde las venas reparadas en el dorso. Si no se obtiene reflujo de sangre, hay que valorar si todavía puede haber lesión intimal o alguna rama traumatizada en la parte amputada. También debe asegurarse que se ha alcanzado un lugar sano para realizar la anastomosis. Si el desbridamiento es amplio debido a una lesión avulsiva, suele necesitarse la interposición de injertos venosos tomados de la cara volar del antebrazo. Ante la duda es preferible utilizar injertos antes que realizar anastomosis a tensión. Los injertos 
venosos deben orientarse en el sentido del flujo vascular a favor de válvulas, es decir que se debe girar el sentido del bypass $180^{\circ}$ en caso de colocarlo en una arteria. Por lo general, la sutura se realiza con hilo de 8/0 o 9/0 montado sobre aguja cilíndrica $3 / 8$ de $75 \mu \mathrm{m}$.

A continuación, se realiza la neurorrafia epineural de los nervios colaterales con hilo 8/0. Si tras la lesión o el desbridamiento se crea un defecto nervioso que no permite la reparación primaria, se puede demorar la interposición de injertos nerviosos a otro tiempo quirúrgico. En aquellos casos en que se disponga de tejido nervioso de dedo banco puede realizarse un injerto nervioso de interposición. En nuestro caso, es extraordinario utilizar dedos bancos ya que nuestra intención es la de reimplantar siempre todos los dedos amputados.

El cierre de heridas se realiza con sutura no absorbible 4/0, al igual que en el dorso, con pocas suturas y poca tensión, para evitar compresión de pedículos. Es preferible no cerrar la heridas y utilizar injertos de piel sin fijar o apósitos de vaselina, antes que un cierre a tensión que comprima las venas. Habitualmente esa parte del procedimiento dura entre 1,5 y 3 horas por dedo.

\section{Cuidados Postquirúrgicos}

Los pacientes sometidos a reimplante deben recibir cuidados en el postoperatorio inmediato en una unidad de reanimación específica. El control postquirúrgico debe incluir control de la temperatura ambiental con lámpara de calor y fluidoterapia de mantenimiento para mantener una tensión arterial sistólica $>110$ mmHg. La posición del paciente durante el postoperatorio es fundamental y debe asegurarse que el codo se apoye cómodamente sobre el colchón, pegado al tronco y suavemente flexionado sin alcanzar ángulo recto, colgado de un palo de gotero (nunca del techo o de la cama), sin estrangular la extremidad, y de forma que el paciente esté confortable ( - Figura 11). Nunca se debe comprimir o cubrir la zona intervenida, y se debe evitar la abducción del hombro. Tampoco se debe calentar la extremidad, especialmente si el paciente permanece inconsciente o si se ha realizado una anestesia regional de larga duración, ya que se podría quemar de forma inadvertida. La vigilancia del estado vascular del reimplante debe realizarse cada 2 horas y el paciente debe permanecer en ayunas hasta la visita médica al día siguiente a la intervención.

En cuanto al tratamiento farmacológico en el postoperatorio inmediato, debe proveerse de analgesia adecuada para su confort. Además, se debe evitar la infección mediante doble antibioterapia con ceftazidima + gentamicina en previsión de contaminación por Pseudomona Aeuriginosa. También debe realizarse profilaxis antitrombótica con heparina de bajo peso molecular, comenzando la primera dosis al terminar la cirugía y extendiendo su administración durante 3 semanas más. El uso de heparina sódica intravenosa o de aspirina no ha demostrado cambios en la supervivencia de nuestros reimplantes. También debe realizarse profilaxis de úlcera péptica.

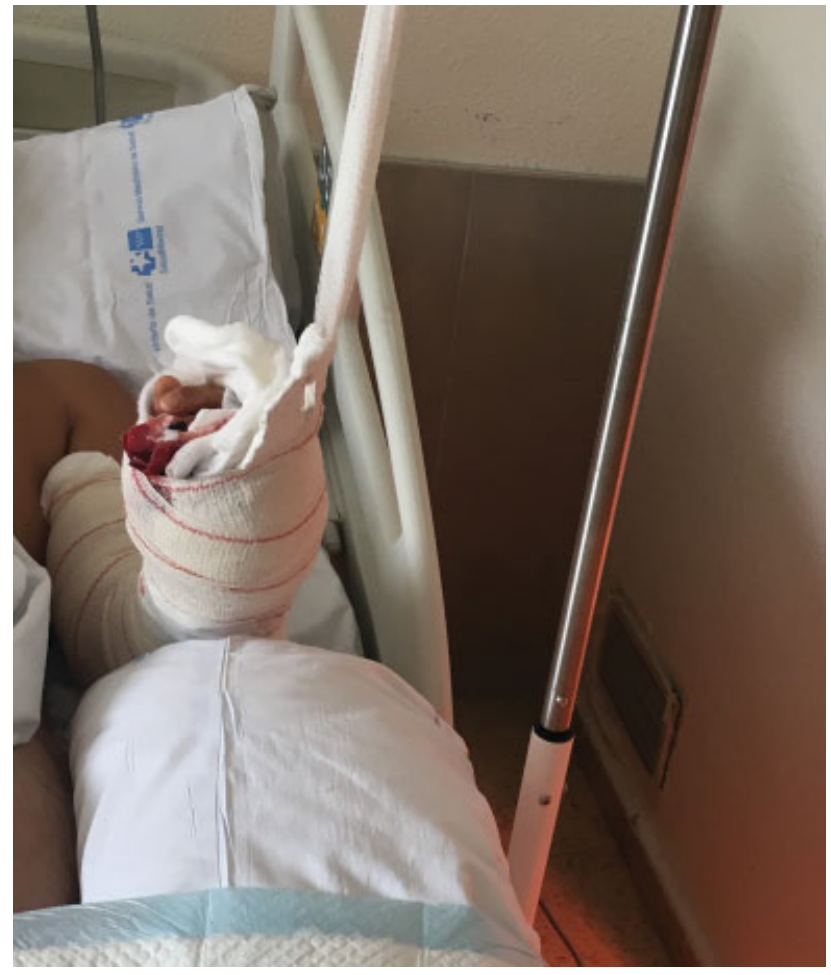

Fig. 11 Medidas posturales de un paciente encamado con punta de dedo reimplantada.

Las sesiones de fisioterapia pueden planificarse según la estabilidad de las osteosíntesis y de las reparaciones tendinosas. La osteosíntesis rígidas con cerclaje 90-90 permiten la movilización antes de las tres semanas postoperatorias. Las agujas de Kirschner retrasan la movilización más allá de las 3 semanas desde el reimplante.

En cuanto a los cambios de curas y la secuencia de retirada de agujas y suturas, en nuestro caso preferimos retrasar los cambios de cura lo máximo que permita la herida, ya que hemos observado en dos ocasiones que el cambio de cura doloroso puede producir un vasoespasmo irrecuperable. De igual manera, las agujas y suturas no se retirarán antes de 3 semanas por riesgo de dolor y vasoespamo. Si hay costras duras y abundantes solemos indicar al paciente medidas higiénicas e hidratación local con vaselina para reblandecer la zona y facilitar la retirada de suturas trascurrido un mes desde la intervención.

\section{Éxito y Medidas de Resultado}

Es importante conocer el porcentaje de éxito en ese tipo de traumatismos y el objetivo funcional que esperaremos según el tipo de lesión. En revascularizaciones nuestro éxito ha sido del $100 \%$, mientras que en reimplantes digitales hemos observado un éxito del 89,5\%, incluyendo reimplante mono y multidigitales, con un porcentaje de revisión urgente del reimplante que alcanza el $7 \%$.

Un porcentaje muy alto de esos pacientes precisarán de cirugías secundarias para llegar al objetivo funcional previsto. ${ }^{14}$ Esas cirugías en su mayoría consisten en tenolisis y artrolisis para la mejora de los rangos de movilidad, artrodesis y osteotomías para corregir la 


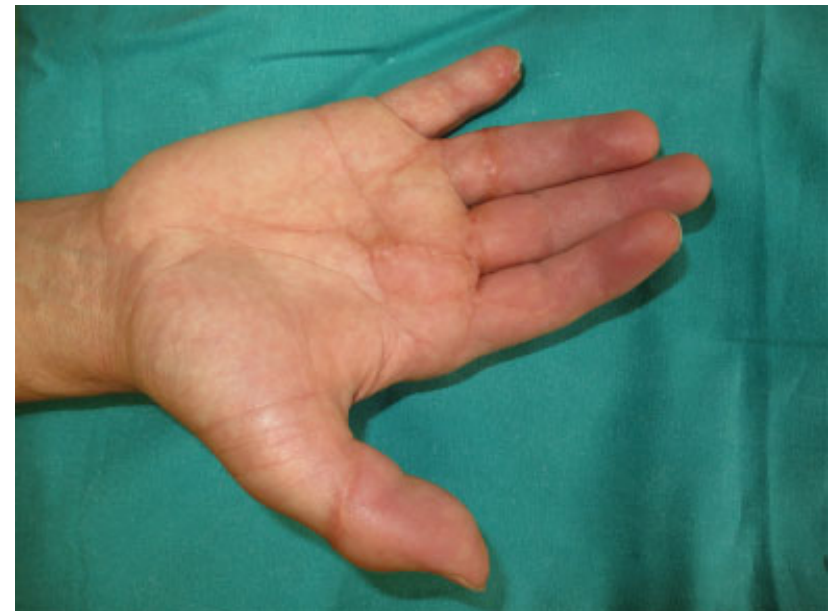

Fig. 12 Resultado de reimplante multidigital en el paciente mostrado en la - Figura 1. Todos los dedos pudieron ser tratados con éxito. El paciente se encuentra pendiente de rehabilitación y cirugía secundaria en función de los déficits funcionales que presente.

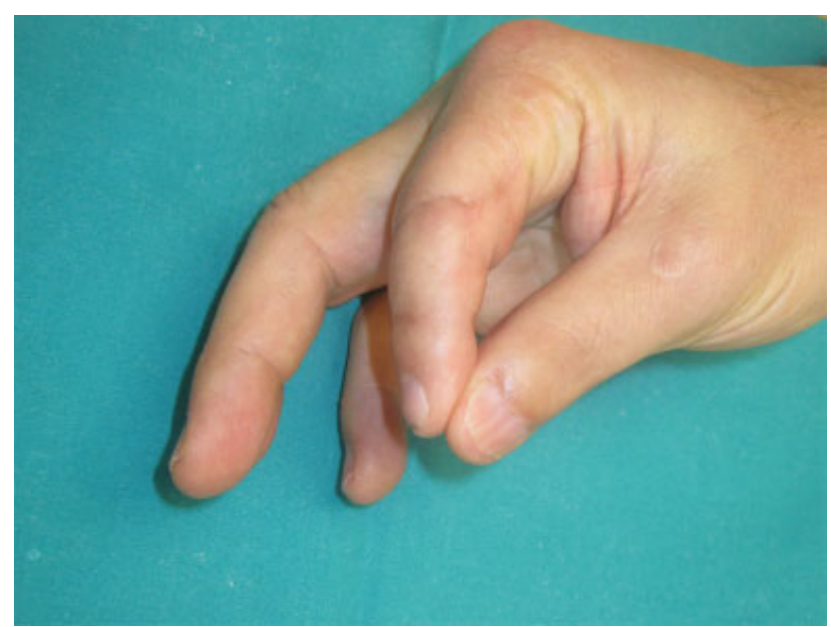

Fig. 13 Resultado de reimplante multidigital del paciente mostrado en la - Figura 5. En el dedo pulgar se realizó reimplante de pulpejo, mientras que el segundo dedo se reimplantó en zona II. Los reimplantes en zona II presentan limitaciones funcionales. En la imagen puede observarse que el paciente realiza una pinza razonable aunque no es capaz de flexionar la articulación interfalángica proximal. Los reimplantes multidigitales tienen indicación de reimplante, incluso aquéllos con amputación en zona II.

posición del dedo, reconstrucción de poleas, injertos de nervio y reconstrucción de tendones en dos tiempos. ${ }^{15}$

El objetivo último del reimplante es la obtención de resultados funcionales satisfactorios a largo plazo, proporcionando a su vez una mano estéticamente aceptable (-Figuras 12 y 13).Una mano con apariencia desagradable que no permita socializar al paciente con normalidad, puede considerarse como un fracaso del reimplante.

\section{Conclusiones}

Los traumatismos que conllevan la amputación de los dedos continúan siendo uno de los mayores retos a los que se debe enfrentar un cirujano de la mano. La ejecución de la anastomosis no es suficiente para obtener el objetivo funcional y estético óptimo. Existen factores en relación al traumatismo, al paciente y al cirujano que pueden alterar gravemente la consecución de esos objetivos.

En este artículo, nuestro objetivo ha sido dar a conocer la correcta consecución de la secuencia del reimplante así como una serie de claves técnicas durante la cirugía del reimplante. Las cirugías de rescate y secundarias, así como la rehabilitación en este tipo de pacientes, si bien fundamentales para el buen resultado del mismo, no han sido objeto directo de discusión en nuestro artículo.

El éxito en la cirugía del reimplante de dedos se sitúa en torno al $90 \%$, con resultados funcionales cada vez mejores. Sin embargo, debemos continuar perfeccionando las diferentes prácticas, disminuyendo así las posibilidades de errores de derivación, de indicación del reimplante, o de la mala ejecución quirúrgica o rehabilitación en ese tipo de pacientes.

\section{Conflicto de Intereses}

Los autores del trabajo declaran no tener ningún conflicto de intereses.

\section{Bibliografía}

1 Marquina P. Reflexiones microquirúrgicas. Rev Iberoam Cir Mano 2015;43(01):1-2

2 Brown M, Lu Y, Chung KC, Mahmoudi E. Annual hospital volume and success of digital replantation. Plast Reconstr Surg 2017;139 (03):672-680

3 Cho HE, Zhong L, Kotsis SV, Chung KC. Finger Replantation Optimization Study (FRONT): update on national trends. JHand Surg Am 2018;43(10):903-912.e1

4 Chung KC, Yoon AP, Malay S, Shauver MJ, Wang L, Kaur S; FRANCHISE Group. Patient-reported and functional outcomes after revision amputation and replantation of digit amputations: The FRANCHISE multicenter international retrospective cohort study. JAMA Surg 2019;154(07):637-646

5 Hustedt JW, Bohl DD, Champagne L. The detrimental effect of decentralization in digital replantation in the United States: 15 Years of evidence from the national inpatient sample. JHand Surg Am 2016;41(05):593-601

6 Pet MA, Morrison SD, Mack JS, et al. Comparison of patient-reported outcomes after traumatic upper extremity amputation: Replantation versus prosthetic rehabilitation. Injury 2016;47(12): 2783-2788

7 Rosales RS, Landin L, Corella F. Hand surgery in Spain. J Hand Surg Eur Vol 2019;44(03):332-334

8 Urbaniak JR, Evans JP, Bright DS. Microvascular management of ring avulsion injuries. J Hand Surg Am 1981;6(01):25-30

9 Yu JC, Shieh SJ, Lee JW, Hsu HY, Chiu HY. Secondary procedures following digital replantation and revascularisation. Br J Plast Surg 2003;56(02):125-128

10 García-Bernal FJ, Zayas P, Regalado J, Julb C. Colgajo microquirúrgico de músculo gracilis para cobertura de palma y dorso de mano tras revascularización. Rev Iberoam Cir Mano 2015;43(01):38-42

11 Adani R, Marcoccio I, Castagnetti C, Tarallo L. Long-term results of replantation for complete ring avulsion amputations. Ann Plast Surg 2003;51(06):564-568, discussion 569 
12 Kwon GD, Ahn BM, Lee JS, Park YG, Chang GW, Ha YC. The effect of patient age on the success rate of digital replantation. Plast Reconstr Surg 2017;139(02):420-426

13 Safa B, Greyson MA, Eberlin KR. Efficiency in replantation/revascularization surgery. Hand Clin 2019;35(02): $131-141$
14 Weinzweig N, Sharzer LA, Starker I. Replantation and revascularization at the transmetacarpal level: long-term functional results. JHand Surg Am 1996;21(05):877-883

15 Landin L, Bolado P, Gajete MA, Gonzalez-Miranda A, Bonastre J. Algorithmic surgical enhancement of function after finger revascularisation. Indian J Plast Surg 2016;49(02):239-244 